\title{
Equalization of Loudspeaker and Room Responses Using Kautz Filters: Direct Least Squares Design
}

\author{
Matti Karjalainen and Tuomas Paatero \\ Department of Electrical and Communications Engineering, Laboratory of Acoustics and Audio Signal Processing, \\ Helsinki University of Technology, P.O. Box 3000, FI 02015, Finland
}

Received 30 April 2006; Revised 4 July 2006; Accepted 16 July 2006

Recommended by Christof Faller

DSP-based correction of loudspeaker and room responses is becoming an important part of improving sound reproduction. Such response equalization (EQ) is based on using a digital filter in cascade with the reproduction channel to counteract the response errors introduced by loudspeakers and room acoustics. Several FIR and IIR filter design techniques have been proposed for equalization purposes. In this paper we investigate Kautz filters, an interesting class of IIR filters, from the point of view of direct least squares EQ design. Kautz filters can be seen as generalizations of FIR filters and their frequency-warped counterparts. They provide a flexible means to obtain desired frequency resolution behavior, which allows low filter orders even for complex corrections. Kautz filters have also the desirable property to avoid inverting dips in transfer function to sharp and long-ringing resonances in the equalizer. Furthermore, the direct least squares design is applicable to nonminimum-phase EQ design and allows using a desired target response. The proposed method is demonstrated by case examples with measured and synthetic loudspeaker and room responses.

Copyright ( 2007 M. Karjalainen and T. Paatero. This is an open access article distributed under the Creative Commons Attribution License, which permits unrestricted use, distribution, and reproduction in any medium, provided the original work is properly cited.

\section{INTRODUCTION}

Equalization of audio reproduction using digital signal processing (DSP), such as improving loudspeaker or combined loudspeaker-room responses, has been studied extensively for more than twenty years [1-8]. Availability of inexpensive DSP processing power almost in any audio system makes it desirable and practical to correct the response properties of analog and acoustic parts by DSP. The task is to improve the system response of a given reproduction channel towards the ideal one, that is, flat frequency response and constant group delay.

It is now commonly understood that this equalization should be done carefully, taking into account physical, signal processing, and particularly psychoacoustic criteria. An ideal equalizer, that is, the inverse filter of a given system response, works only in offline simulations [6]. Even for a point-to-point reproduction path, minor nonstationarity of the path and limitations in response measurement accuracy make ideal equalization impossible. Furthermore, monophonic reproduction has to be usually considered as a SIMO (single-input multiple-output) system since the signal may be received in different points, whereas multichannel reproduction is correspondingly a MIMO (multiple-input multiple-output) system. However, in this paper we restrict ourselves to study point-to-point reproduction paths only.

The problem of loudspeaker response equalization is simpler than the correction of a full acoustic path including room acoustics. Loudspeaker impulse responses are relatively short and the magnitude response is regular in a welldesigned speaker. EQ filter techniques proposed for the purpose include FIR filters, warped FIR and IIR filters [2], and Kautz filters [9]. FIR filters are straightforward to design but require using high orders because of the inherently uniform frequency resolution that is highly nonoptimal at lowest frequencies. Furthermore, long FIR equalizers may produce pre-echo problems, that is, audible signal components arrive before the main response. Warped and Kautz filters allocate frequency resolution better, thus reducing required filter orders radically. Flattening of loudspeaker magnitude response on the main axis to inaudible deviations can be done quite easily with any of these techniques. For a highquality speaker the phase response errors (group delay deviations) are often not perceivable without any correction, 
but nonminimum-phase EQ designs can improve this even further. A particular advantage of DSP-based loudspeaker equalization is that the design of the speaker itself can be optimized by other criteria, while good final response characteristics are obtained by DSP.

Room response equalization is a much harder problem than improving loudspeaker responses only. From a filter design point of view, the same FIR and IIR techniques as in loudspeaker equalization are available for room response correction, but depending on the case, filter orders become much higher.

While flattening of the magnitude response also in this case is relatively easy to carry out, difficult problems are found particularly in reducing excessive reverberation, reflections from room surfaces, and sharp resonances due to low-frequency room modes. Reduction of the effect of perceived room reverberation, in order to improve clarity, is a very hard task because of the highly complex modal behavior of rooms at mid to high frequencies. By proper shaping of the temporal envelope of the response, for example, by complex smoothing technique in EQ FIR filter design $[10,11]$, this can be achieved to some degree. This requires necessarily high-order equalization filters. Counteracting room surface reflections is only possible to a specified point in the space, from where the receiver is allowed to move less than a fraction of wavelength of the highest frequency in question. At lowest frequencies, modal equalization [12] has been developed to control the temporal decay characteristics of modal resonances that have too high Q-values.

In all cases of EQ filter design the basic problem is to select and realize a filter structure and then to calibrate it at the site of audio reproduction. This reminds adaptive filtering although the adaptation in most cases is done only offline and kept fixed as far as no recalibration is required. From the viewpoint of this paper we divide the filter parameter estimation techniques into two categories. Figure 1(a) shows a case where the EQ filter target response is obtained separately by any appropriate response inversion method, after which the EQ filter is optimized to approximate that with given criteria. We call this the indirect design approach. Figure 1(b) depicts the direct method where the difference between desired and equalized response is minimized directly in the least squares (LS) sense in the EQ filter calibration process.

Another conceptual categorization for the purpose of this paper is the division to minimum-phase and nonminimumphase equalization. Minimum-phase inversion of the measured response is often applied because of simplicity, after which the EQ filter is designed to approximate this minimum-phase part of the equalizer target response. That means correcting only the magnitude response, while nonminimum-phase characteristics remain as they are. This is enough in most loudspeaker equalization tasks as well as in basic room response correction, but certain EQ tasks require nonminimum-phase processing.

Based on these categorizations we can now characterize different equalization filter design methods. Direct inversion in the transform domain through discrete Fourier transform,

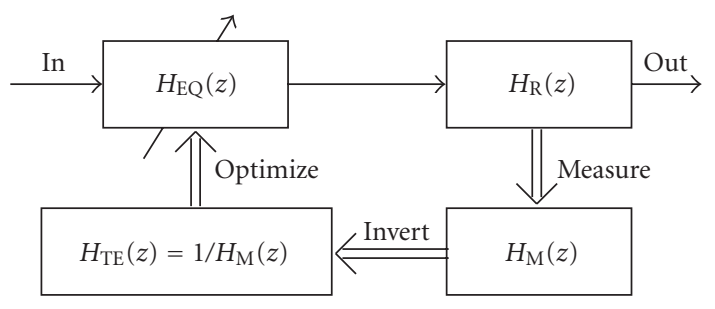

(a)

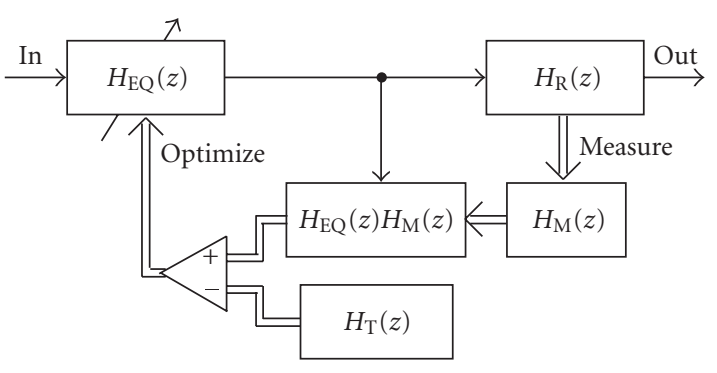

(b)

FIgure 1: (a) Indirect and (b) direct EQ filter design. $H_{\mathrm{EQ}}(z)$ is equalization filter, $H_{\mathrm{R}}(z)$ is reproduction channel, $H_{\mathrm{M}}(z)$ is measured response. Target response denotations $H_{\mathrm{TE}}(z)$ and $H_{\mathrm{T}}(z)$ distinguish between the two different equalization configurations. Audio signals are denoted by single line and filter design data by double line.

that is, $H_{\mathrm{EQ}}(z)=1 / H_{\mathrm{M}}(z)$ in Figure $1(\mathrm{a})$, is problematic in many ways and cannot be used directly $[6,13]$, so that some modifications have to be applied to obtain useful results. These methods may apply some preprocessing such as complex smoothing before inversion to obtain $H_{\mathrm{EQ}}(z)$.

A direct method for obtaining an FIR equalizer is AR modeling (linear prediction) of $H_{\mathrm{M}}(z)$ to get an all-pole filter, the inverse of which is an FIR filter for $H_{\mathrm{EQ}}(z)$ [2]. The method results in minimum-phase equalization. This approach allows also to realize warped FIR filters when using proper prewarping before AR modeling [2]. In warped IIR design [2] the measured response is first minimum-phase inverted and prewraped and then ARMA (pole-zero) modeled, thus belonging primarily to the category of indirect modeling. In [9], Kautz filters have been used in a similar indirect way but with increased freedom of allocating frequency resolution. The direct LS design of Kautz equalizers was suggested for the first time in [14]. In the present paper we generalize and expand this approach.

The rest of this paper is structured as follows. Section 2 introduces the concept of Kautz filters. Section 3 presents the principles of Kautz modeling and EQ filter design, including both LS design of tap coefficients and principles for Kautz pole selection. Loudspeaker equalization cases are studied in Section 4 and room response correction is investigated in Section 5. This is followed by discussion and conclusions.

\section{KAUTZ FILTERS}

The Kautz filter has established its name due to a rediscovery in the early signal processing literature $[15,16]$ of an even 


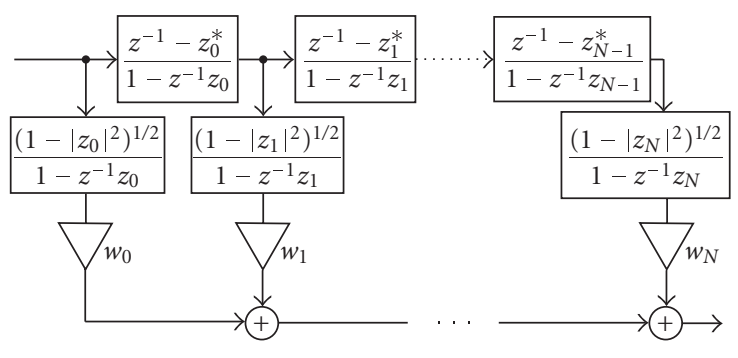

FIgURE 2: The Kautz filter. For $z_{i}=0$ in (1) it degenerates to an FIR filter, for $z_{i}=a,-1<a<1$, it is a Laguerre filter where the tap filters can be replaced by a common prefilter.

older mathematical concept related to rational representations and approximations of functions [17]. The generic form of a Kautz filter is given by the transfer function

$$
\begin{aligned}
H(z) & =\sum_{i=0}^{N} w_{i} G_{i}(z) \\
& =\sum_{i=0}^{N} w_{i}\left(\frac{\sqrt{1-z_{i} z_{i}^{*}}}{1-z_{i} z^{-1}} \prod_{j=0}^{i-1} \frac{z^{-1}-z_{j}^{*}}{1-z_{j} z^{-1}}\right),
\end{aligned}
$$

where $w_{i}, i=0, \ldots, N$, are somehow assigned tap-output weights. The orthonormal Kautz functions $G_{i}(z), i=$ $0, \ldots, N$, are determined by any chosen set of stable poles: $\left\{z_{j}\right\}_{j=0}^{N}$, such that $\left|z_{j}\right|<1$. The superscript $(\cdot)^{*}$ denotes complex conjugation. Figure 2 may be a more instructive description than formula (1).

Defined in this manner, Kautz filters are merely a class of fixed-pole IIR filters that are forced to produce orthonormal tap-output impulse responses. However, a Kautz filter is in fact more genuinely a generalization of the FIR filter and its warped counterparts, which is characterized in terms of properties of the all pass filter that constitutes the backbone of a tapped transversal structure in Figure 2.

It is easy to see that if $z_{j}=0$ for all $j$, the Kautz structure is reduced to an FIR filter. For $z_{j}=a$, a fixed value $-1<a<1$ for all $j$, a Laguerre filter is obtained.

The time-domain counterpart of (1), the Kautz filter impulse response, is given by

$$
h(n)=\sum_{i=0}^{N} w_{i} g_{i}(n)
$$

where functions $\left\{g_{i}(n)\right\}_{i=0}^{N}$ are impulse responses or inverse $z$-transforms of functions $\left\{G_{i}(z)\right\}_{i=0}^{N}$. The meaning of orthonormality is specified most economically by defining the time-domain inner product of two (causal) signals $x(n)$ and $y(n)$,

$$
\langle x, y\rangle:=\sum_{n=0}^{\infty} x(n) y^{*}(n)
$$

Now, impulse responses $\left\{g_{i}(n)\right\}_{i=0}^{N}$ are orthogonal in the sense that $\left\langle g_{i}, g_{k}\right\rangle=0$ for $i \neq k$, and normal, since $\left\langle g_{i}, g_{i}\right\rangle=1$ for $i=0, \ldots, N$.
A reasonable presumption in modeling a real response is that the poles $z_{j}$ should be real or occur in complexconjugate pairs. For complex-conjugate poles, an equivalent real Kautz filter formulation [15], depicted in Figure 3, prevents dealing with complex (internal) signals and filter weights. The normalization terms in the real Kautz structure are

$$
\begin{aligned}
& p_{i}=\sqrt{\frac{\left(1-\rho_{i}\right)\left(1+\rho_{i}-\gamma_{i}\right)}{2}}, \\
& q_{i}=\sqrt{\frac{\left(1-\rho_{i}\right)\left(1+\rho_{i}+\gamma_{i}\right)}{2}},
\end{aligned}
$$

where $\gamma_{i}=-2 \operatorname{RE}\left\{z_{i}\right\}$ and $\rho_{i}=\left|z_{i}\right|^{2}$ are expanded polynomial coefficients of the second-order blocks. The all pass characteristics of the transversal blocks are restored by shifting the denominators in Figure 3 one step to the right and by compensating for the change in the tap-output blocks. A mixture of structures in Figures 2 and 3 is used in the case of both real and complex-conjugate poles.

\section{MODELING AND EQUALIZATION USING KAUTZ FILTERS}

There are two different aspects of optimization when using Kautz filters in system modeling and equalization: (a) finding optimal tap coefficients $\left\{w_{i}\right\}$ and (b) finding an optimal set of Kautz poles $\left\{z_{j}\right\}$. The former problem can be solved as an LS problem, while finding optimal poles (together with tap coefficients) is necessarily an iterative or a search process.

In this section we first study the former problem. That is, modeling and equalization of system responses when there is a prefixed set of Kautz poles. Modeling of a given $H_{\mathrm{TE}}(z)$ is discussed first briefly and the main topic, direct LS EQ design, then in more detail. Thereafter the selection of Kautz poles, that is, allocation of frequency resolution, is studied.

\subsection{Kautz modeling of a given response}

When an equalizer target response $h_{\mathrm{TE}}(n)$ for "forward modeling" is given, the task of approximating it by a Kautz filter is particularly straightforward: a desired pole set is selected to form the basis functions $g_{i}(n)$, after which the approximation is composed as

$$
h_{\mathrm{EQ}}(n)=\sum_{i=0}^{N} c_{i} g_{i}(n), \quad c_{i}=\left\langle h_{\mathrm{TE}}, g_{i}\right\rangle,
$$

that is, the filter weights $c_{i}$ are the orthogonal expansion coefficients (Kautz-Fourier coefficients) of $h_{\mathrm{TE}}(n)$ with respect to the choice of the basis functions.

One of the favorable specialities of Kautz filter design, compared to other IIR or pole-zero filter configurations, is that the approximation is independent of rearrangement of the pole set, which implies means for reducing as well as extending the model by pruning, tuning, and appending poles, respectively. In addition, the use of orthogonal expansion coefficients corresponds to LS design with respect to the particular pole set, and as a consequence of the orthogonality, the 


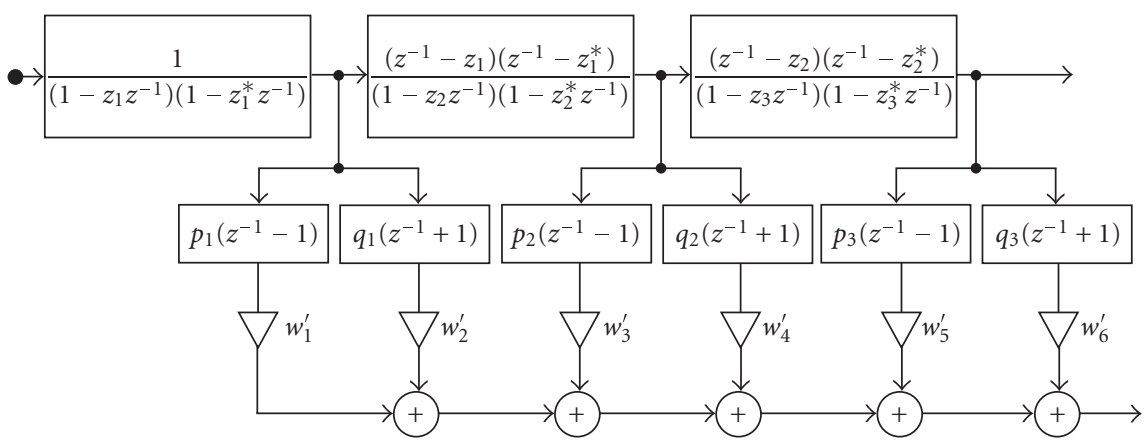

FIGURE 3: One possible realization of a real Kautz filter, corresponding to a sequence of complex-conjugate pole pairs [15].

approximation error (energy) $E$ is given simply as

$$
E=E_{\mathrm{TE}}-\sum_{i=0}^{N} c_{i}^{2}
$$

where $E_{\mathrm{TE}}$ is the energy of the target response. As an alternative to the evaluation of $c_{i}=\left\langle h_{\mathrm{TE}}, g_{i}\right\rangle$ using the inner product formula (3), the Kautz filter tap-output weights are also obtained by feeding the signal $h_{\mathrm{TE}}(-n)$ to the Kautz filter and reading the tap outputs $x_{i}(n)$ at $n=0: c_{i}=x_{i}(0)$. That is, all inner products in (5) are implemented simultaneously using filtering. Note that in the case of an FIR filter this would equal the design by truncation of $h_{\mathrm{TE}}(n)$.

The "forward modeling" approach was applied in [9] according to the indirect method of Figure 1(a) by first minimum-phase inverting a measured impulse response and then applying the Kautz modeling. Theoretically another way is to make a Kautz model directly for the measured response and try to invert it, which is however problematic because the nonminimum-phase model leads to an unstable filter. In fact, this kind of inversion schemes are particularly unattractive from the point of view of Kautz filters because of the numerator configuration in the transfer function.

\subsection{Direct LS equalization using Kautz filters}

The equalization method that is of main interest in this paper is the direct EQ configuration by least squares Kautz filter design as shown in Figure 1(b). The equalizer, with impulse response $h_{\mathrm{EQ}}(n)$, is identified in cascade with the system $h_{\mathrm{R}}(n)$ based on measurement $h_{\mathrm{M}}(n)$ in order to approximate the target response $h_{\mathrm{T}}(n)$ in the time-domain by

$$
h_{\mathrm{E}}(n)=h_{\mathrm{EQ}}(n) * h_{\mathrm{R}}(n) \approx h_{\mathrm{T}}(n),
$$

where $(*)$ is the convolution operator. The direct equalization is provided by the least squares configuration [18]: the square error in the approximation (7) is minimized with respect to the equalizer parameters (filter tap coefficients). In terms of the Kautz equalizer, the tap-output weights $\left\{w_{i}\right\}$ are optimized according to

$$
\min _{w_{i}}\left(\sum_{n}\left(h_{\mathrm{E}}(n)-h_{\mathrm{T}}(n)\right)^{2}\right),
$$

where the equalized response

$$
h_{\mathrm{E}}(n)=\sum_{i=0}^{N} w_{i} x_{i}(n), \quad x_{i}(n)=g_{i}(n) * h_{\mathrm{R}}(n) .
$$

Using system identification terminology, the equalization setup is an output-error configuration with respect to a special choice of model structure. It can even be considered as a generalized linear prediction: we could call it "Kautz prediction." Furthermore, it is a quadratic LS problem with a well-defined and unique solution that is obtained from the corresponding normal equations. If the Kautz equalizer tapoutput responses $x_{i}(n)=g_{i}(n) * h_{\mathrm{R}}(n)$ are assembled into a "generalized channel convolution matrix"

$$
\mathbf{S}=\left[\begin{array}{ccc}
x_{0}(0) & \cdots & x_{N}(0) \\
x_{0}(1) & \cdots & x_{N}(1) \\
\vdots & \ddots & \vdots \\
x_{0}(L) & \cdots & x_{N}(L)
\end{array}\right],
$$

then the normal equations submit to the matrix form

$$
\mathbf{S}^{T} \mathbf{S w}=\mathbf{s}, \quad \mathbf{w}=\left[\begin{array}{lll}
w_{0} & \cdots & w_{N}
\end{array}\right]^{T},
$$

where $\mathbf{s}$ is the (cross-)correlation vector between the tapoutput responses and the desired target response $h_{\mathrm{T}}(n), s_{i}=$ $\left\langle h_{\mathrm{T}}, x_{i}\right\rangle$. The matrix product $\mathbf{S}^{T} \mathbf{S}$, where $(\cdot)^{T}$ denotes transpose of a matrix, implements correlation analysis of the tapoutput responses, $\left\langle x_{i}, x_{j}\right\rangle$, in terms of the inner product (3), where it is presumed that the Kautz filter responses are realvalued. Here we consider only the case of an impulse as the target response, $h_{\mathrm{T}}(n)=\delta(n-\Delta)$, where $\delta(\cdot)$ is the unit impulse, including a potential delay $\Delta$. Then the correlation vector simply picks the $(\Delta+1)$ th row of the matrix $\mathbf{S}$,

$$
\mathbf{s}=\left[\begin{array}{lll}
x_{0}(\Delta) & \cdots & x_{N}(\Delta)
\end{array}\right]^{T} .
$$

The solution of the matrix equation (11) is

$$
\mathbf{w}=\left(\mathbf{S}^{T} \mathbf{S}\right)^{-1} \mathbf{S}
$$

and it provides the LS optimal equalizer tap-output weights with respect to the choice of Kautz functions $g_{i}(n)$. 
A specialized question is the choice of the "correlation length" $L$. Our choice is to use a sufficiently large $L>M$, where $M$ is the (effective) length of the response $h_{\mathrm{M}}(n)$, that in practice drains out the memory of the Kautz equalizer for $h_{\mathrm{M}}(n)$. For a particular choice of a Kautz filter this length could also be quantized since the Kautz filter response is a superposition of decaying exponential components. This is in fact not a big issue due to the nature of the configuration, and in practice any $L>M$ will collect the essential part of the "correlation energy," for example, the choice $L=M+N$ as in the conventional LS setting.

\subsection{Selection of Kautz poles and frequency resolution}

Full optimization of an equalizer filter could be defined as finding the lowest (or low enough) order filter that meets the required response quality criteria and other criteria such as stability and numerical robustness. For Kautz filters this includes optimizing both the tap coefficients and the pole positions. As with IIR filters in general, optimizing poles is a complex task.

In Kautz filters, due to the orthonormality of the polerelated subsections, there is an interesting interpretation for pole positioning. Inspired by frequency-warped filters [19], in [9] we have used the negated phase function of the Kautz all pass backbone as a frequency mapping and the negated phase derivative as a function to characterize the inherent allocation of frequency resolution induced by pole positions. This implies that when high resolution is needed around a certain frequency, there should be a pole near the corresponding angle and close to the unit circle. The relationship between the all pass operator and the corresponding orthonormal filter structure (the Kautz filter) is explained more thoroughly in [9]. Several resolution allocation strategies are discussed briefly below and within case examples.

\subsection{Approximation of log-scale resolution}

The logarithmic frequency scale is the most natural one in audio technology due to the nearly logarithmic ERB scale [20] corresponding to the resolution of the human auditory system. The desired log-like frequency resolution ${ }^{1}$ is produced simply by choosing the Kautz filter poles according to a logarithmically spaced pole distribution. In polar coordinates, a set of poles

$$
\left\{z_{1}, \ldots, z_{N}\right\} \rightleftharpoons\left\{r_{1} e^{j \omega_{1}}, \ldots, r_{N} e^{j \omega_{N}}\right\}
$$

is generated, where the angles $\left\{\omega_{1}, \ldots, \omega_{N}\right\}$ correspond to logarithmic spacing for a chosen number of points between 0 and $\pi$. We choose the corresponding pole radius as an exponentially decreasing sequence

$$
r_{i}=\alpha^{\omega_{i}}, \quad \alpha=e^{\ln \left(r_{1}\right) / w_{1}}, \quad r_{1}<1
$$

\footnotetext{
${ }^{1}$ Parallel all pass structures have also been proposed to obtain logarithmic resolution scaling [21].
}

This choice of pole radii will provide an approximately constant-Q resolution for the Kautz equalizer. Each pole is then "duplicated" with its complex-conjugate to produce a real Kautz filter (Figure 3). From a practical point of view, the poles are generated using the formulas

$$
\begin{gathered}
\omega_{i}=\frac{2 \pi f_{i}}{f_{\mathrm{s}}}, \\
p_{i}=R^{\omega_{i} / \pi} e^{ \pm j \omega_{i}},
\end{gathered}
$$

where $p_{i}$ is the $i$ th pole pair $\left\{z_{i}, z_{i}^{*}\right\}, f_{i}$ is the corresponding frequency (in $\mathrm{Hz}$ ), $R$ is the pole radius corresponding to the Nyquist frequency $f_{\mathrm{s}} / 2$, and $f_{\mathrm{s}}$ is the sample rate (in $\mathrm{Hz}$ ).

Figure 4 characterizes the phase and resolution behavior of a log-scale Kautz filter when the pole radii of a spirallike set of complex-conjugate poles are varied, as shown in the $z$-domain pole plot in Figure 4(a). The all pass phase and its derivative are plotted with different scales in subplots (Figures 4(b)-4(d)). With small values of pole radii the phase derivative (resolution function) is smooth and approximately linear on a log-log scale (Figure 4(d)), while with poles closer to the unit circle the phase derivative shows a peak for each pole frequency.

The resolution behavior is also seen in the magnitude spectra of real Kautz filter tap outputs, as plotted for a selected set of log-scaled poles in Figure 5. The constant-Q behavior can be easily observed. Each pole pair generates a pair of orthogonal outputs with the corresponding resonance frequencies and equal Q-values. The sum of the magnitude spectra also characterizes the resolution function of the Kautz filter. A rule of thumb for obtaining a smooth resolution function is to set the neighboring resonance curves to cross each other at approximately $-3 \mathrm{~dB}$ points. As the case studies below show, the selection of pole radii is often not critical at all.

\subsection{Iterative pole positioning techniques}

Iterative methods, such as Prony's method [22] and the Steiglitz-McBride method [23], are common in IIR filter design. For Kautz filters we have successfully applied what we call the BU-method to iteratively search for an optimal positioning of Kautz poles.

The BU-method is based on an old concept of complementary signals [24] that relates the optimization problem of an orthonormal rational filter structure (the Kautz filter) to the properties of the all pass part of the filter. The orthogonal nature of the approximation error induced by a chosen Kautz filter representation was presented in Section 3.1. In addition, a practical method for the evaluation of the filter coefficients was given: if the time-inverted target signal $h(-n), M, \ldots, 0$, is fed to the chosen Kautz filter, then the LS optimal filter weights are attained as the tap-output samples at $n=0$. The optimization problem with respect to the poles can thus be seen as an energy compaction procedure: how to choose the poles so that the energy (sum of squares) of the filter weights is maximized. The "principle of complementary signals" [24] now states that an equivalent objective is to minimize the energy of the all pass filter response $a(n)=$ 


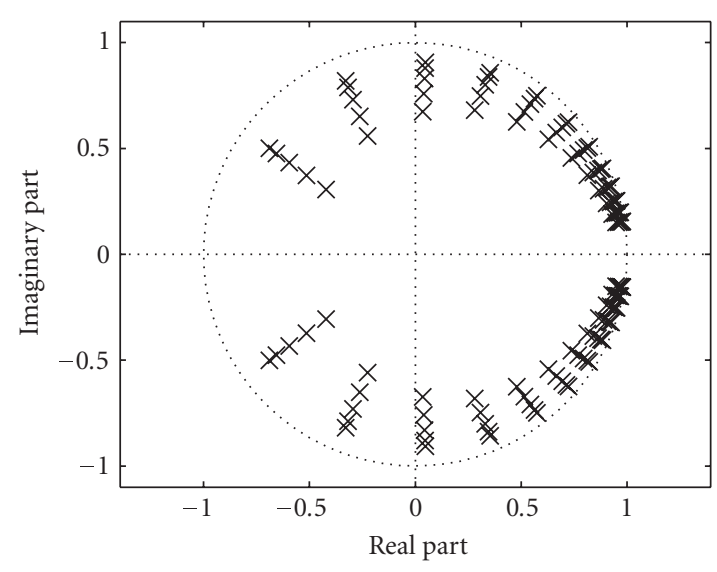

(a)

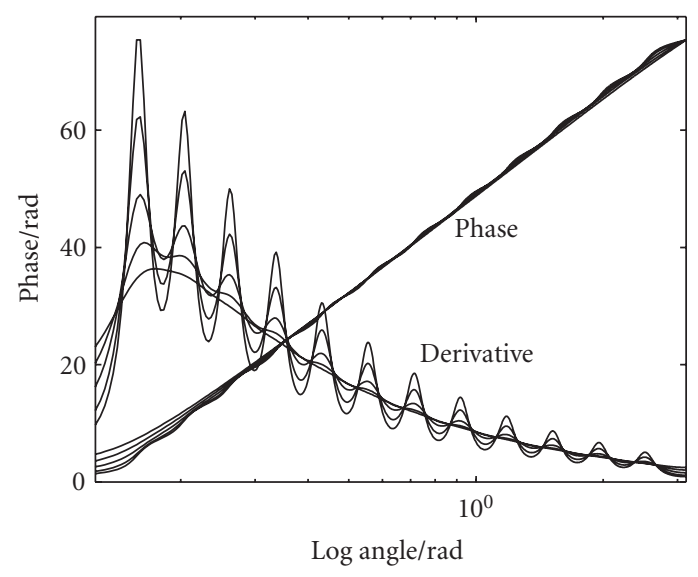

(c)

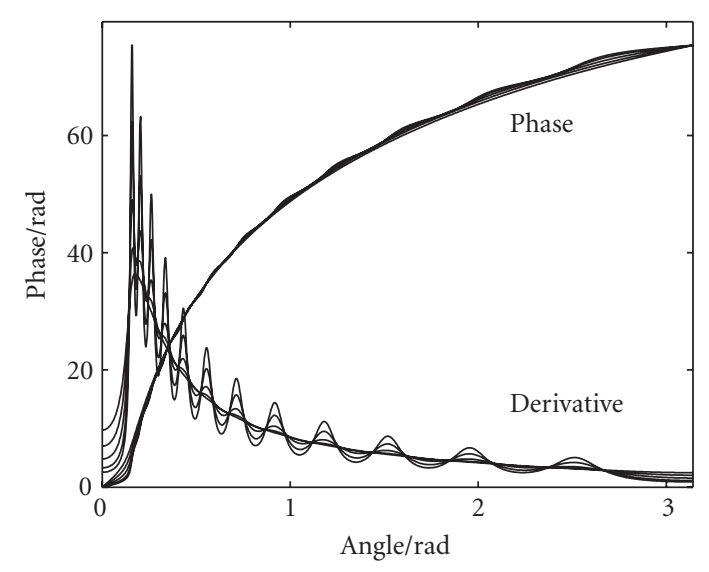

(b)

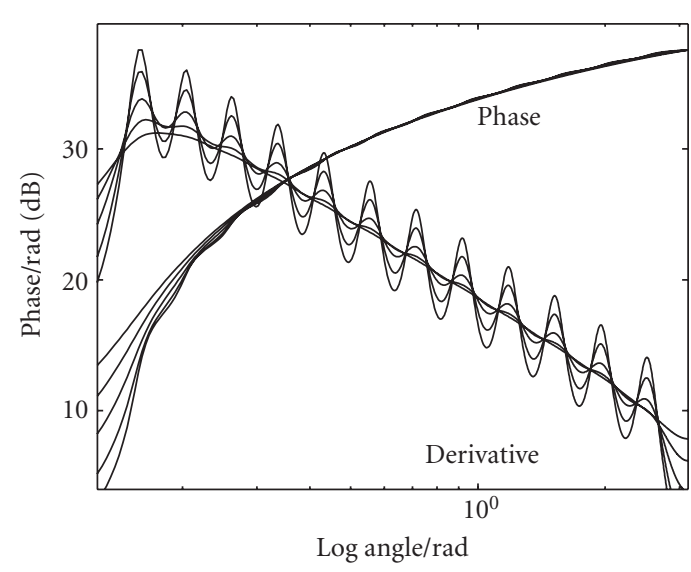

(d)

FIGURE 4: All pass filter characteristics for varying pole radius damping: (a) pole sets; (b) phase functions and phase derivatives; (c) on log-scale; and (d) in $\mathrm{dB}$ on log-scale.

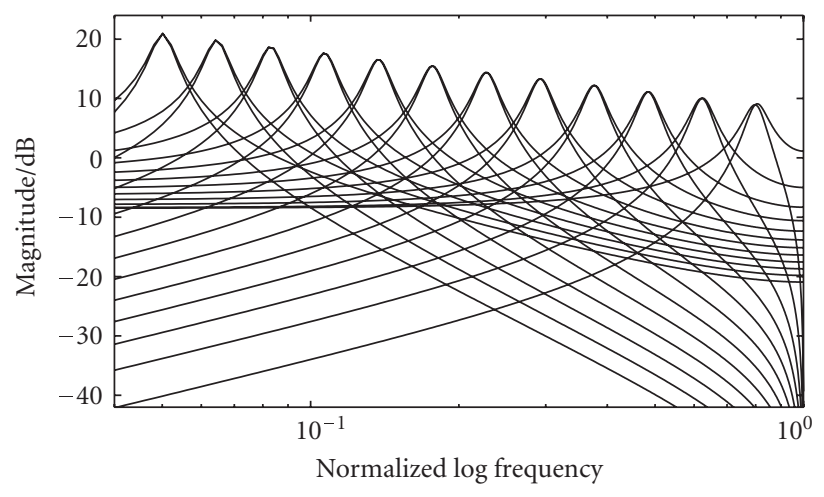

FIgURE 5: Magnitude responses of the Kautz filter tap-output impulse responses with respect to logarithmic distribution of poles.

$A[h(-n)]$ in the interval $[-M, 0]$, where $A(z)$ is the transversal all pass part of the Kautz filter. For the optimization of the all pass filter we have utilized an iterative procedure proposed by Brandenstein and Unbehauen [25], which explains our choice of naming the BU-method.

The BU-method has been applied successfully together with frequency warping to obtain perceptually relevant allocation of frequency resolution. It should be emphasized that here the utilization of the method to optimize Kautz equalizer poles is based on an estimate of the response $H_{\mathrm{TE}}(z)=$ $1 / H_{\mathrm{M}}(z)$. Further details on the BU-method are out of the scope of this paper, they can be found in $[9,26]$.

\subsection{Other pole positioning strategies}

Information about the system to be equalized, whether from measured response or known otherwise, can be used to help in the selection of good pole positions. AR modeling (linear prediction) can be applied to find a good initial set of system poles, or variation in power spectrum is analyzed to find the need for equalization resolution as a function of frequency. 


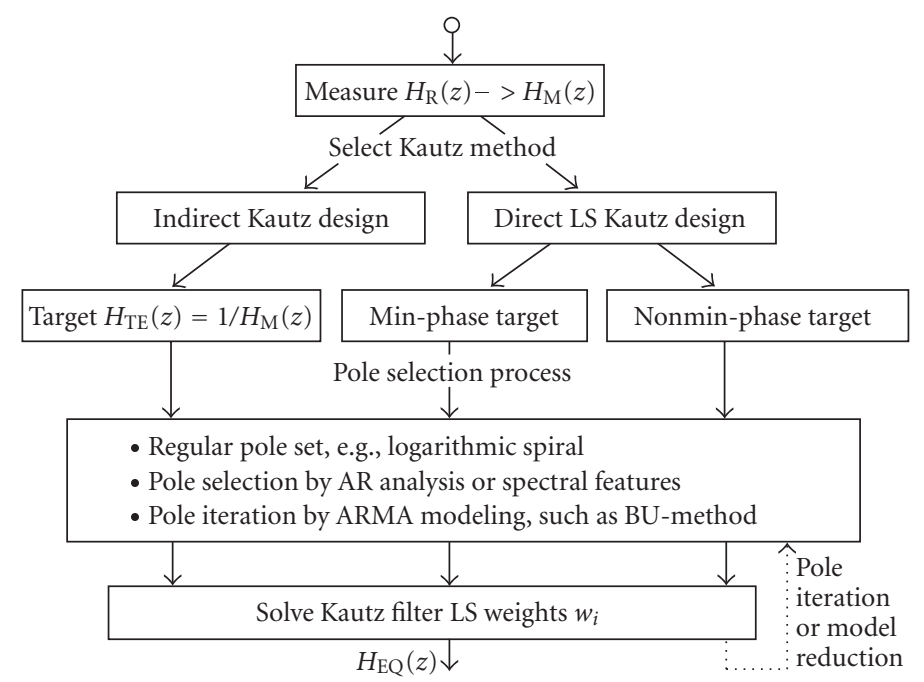

Figure 6: Flow diagram of Kautz filter equalizer design for a set of different methods.

Advanced search techniques such as genetic algorithms may be useful if no side information is available about potential pole positioning although this may require excessive time of computation. Notice that when searching for the lowest filter order to meet given criteria, the filter order is also one of the variables to be iterated.

Hand tuning by an experienced designer may also lead to a good final EQ filter, for example, by discarding or inserting poles in strategic positions.

\subsection{Specification of equalization target}

There are some important topics to be kept in mind when selecting the target response of equalization. Here we emphasize two of them: delay of the response onset and compensation for the roll-offs of loudspeaker response.

In direct LS equalizer design it is possible to set a desired target response, which normally is a unit impulse. If it corresponds to zero time delay, a minimum-phase EQ filter is obtained. By delaying the target impulse more than the maximum group delay of the measured response, see (12), the equalization process starts to correct the phase behavior also. In such a case it is desirable to include an FIR part (i.e., poles at the origin) about the size of the measured group delay or more, as will we discussed in the case studies below.

Figure 6 shows a flow diagram of Kautz filter equalizer design for a set of different methods at each step of the design process.

\section{LOUDSPEAKER EQUALIZATION CASES}

In this section we discuss three cases of loudspeaker equalization, first focusing on magnitude correction and then including phase correction by using nonminimum-phase EQ filter design.

Loudspeakers are typically designed to deal with high signal levels with low distortion only within their pass-band.
The low- and high-frequency roll-offs should therefore not be flattened away although it is computationally possible. In most cases a good choice is to keep these roll-offs as they behave naturally. For example, the low cut-off highpass is of fourth order for a bass reflex design and of second order for a closed box design. A simple way to take these into account is to inverse-compensate the measured response according to these rules, or otherwise straighten it beyond roll-off frequencies. Hence the equalizer designed with this target keeps the natural roll-offs of the loudspeaker response.

\subsection{Loudspeaker equalization, Case 1}

The first example of Kautz equalizer design is presented in Figure 7. It is based on a measured loudspeaker response that has a relatively nonflat magnitude response (Curve (a)). The response is corrected by a 24 th-order ( 12 pole pairs) Kautz filter with logarithmically positioned pole frequencies between $80 \mathrm{~Hz}$ and $23 \mathrm{kHz}$ (indicated by vertical lines in the middle of the figure) and $R=0.03$ (see (15b)). After lowand high-frequency roll-off compensations to avoid boosting off-bands of the speaker, as shown by Curve (c), the EQ filter resulting from Kautz LS equalization has the magnitude response of Curve (d). The equalized response is plotted in Curve (e) and as a 1/3-octave smoothed version in Curve (f).

Filter orders from 8 up (4 pole pairs) give useful results in this case although the selection of order and pole positions may introduce considerable variation in flatness of the result. Therefore full optimization requires a search over sets of poles and filter orders, in spite of the fact that the LS procedure itself always gives optimal tap coefficients for a given fixed order and pole set.

Curve (g) in Figure 7 demonstrates the effect of poor Kautz pole radius selection. In this case the poles are set too close to the unit circle $(R=0.8)$, thus the frequency ranges around the pole frequencies get too much emphasized. 
Otherwise, in most cases, the selection of pole radii is not critical at all. Even very small radii, such as $R=10^{-5}$, work well in this case.

Comparison of Curves (e) and (g) explains also clearly why LS equalization using the Kautz filter configuration can be controlled to behave favorably with dips in the response to be equalized, while exact inversion of a response with deep dips results in undesirable peaks and long-ringing decay times in the equalizer [10]. In Kautz filters the pole radii determine the maximum Q-values of resonances. If pole radii are selected conservatively, no excess peaking and ringing of resonances appear in the equalizer response.

\subsection{Loudspeaker equalization, Case 2}

In the second example of Kautz EQ filter design, both the direct and indirect methods are investigated using the measured response of Case 1. The Kautz filter poles are generated in both cases using a warped counterpart of the BU-method [27] with respect to the inverted target response. The equalizer filter order is chosen to be 38 (18 complexconjugate pole pairs and two real poles). The purpose of this example is to demonstrate that two very different equalizer parametrization schemes, corresponding to (5) and (9), respectively, produce very similar magnitude response correction results, as depicted in Figure 8. The original response, the equalized ones, and the equalizer responses are shown, as well as the pole frequencies obtained from the BU-method. Notice that the poles are allocated mostly to areas where the need for correction is highest. ${ }^{2}$

The ability of the direct LS method to improve phase characteristics is demonstrated in Figure 9. The early part of the measured loudspeaker impulse response and the minimum-phase LS equalized response are displayed in panels (a) and (b). In panel (c) the LS equalizer is designed with respect to a delay $\Delta=12$ samples in the target of equalization. The pole set that is generated from a minimumphase target response is not very good at producing pure delay components, which results also in inefficiency in magnitude equalization (not shown). A way to obtain better equalization is to include zeros in the Kautz filter pole set: in Figure $9(\mathrm{~d})$ the equalizer is equipped with 12 additional poles at the origin, that is, part of the Kautz filter is implemented as an FIR filter substructure. As can be seen, the equalized response is closer to pure impulse (with the additional delay) than in panels (a)-(c), which means more uniform group delay.

\subsection{Loudspeaker equalization, Case 3}

To gain more insight over the nonminimum-phase equalization, that is, of both magnitude and phase, it is advantageous to demonstrate the phase correction by using a synthetic (simulated) loudspeaker response instead of a real measured one. Figures 10 and 11 depict the magnitude and group delay

\footnotetext{
2 From a practical point of view, the correction of sharp peaks and dips in loudspeaker response is not needed and may even worsen the result in directions off from the main axis.
}

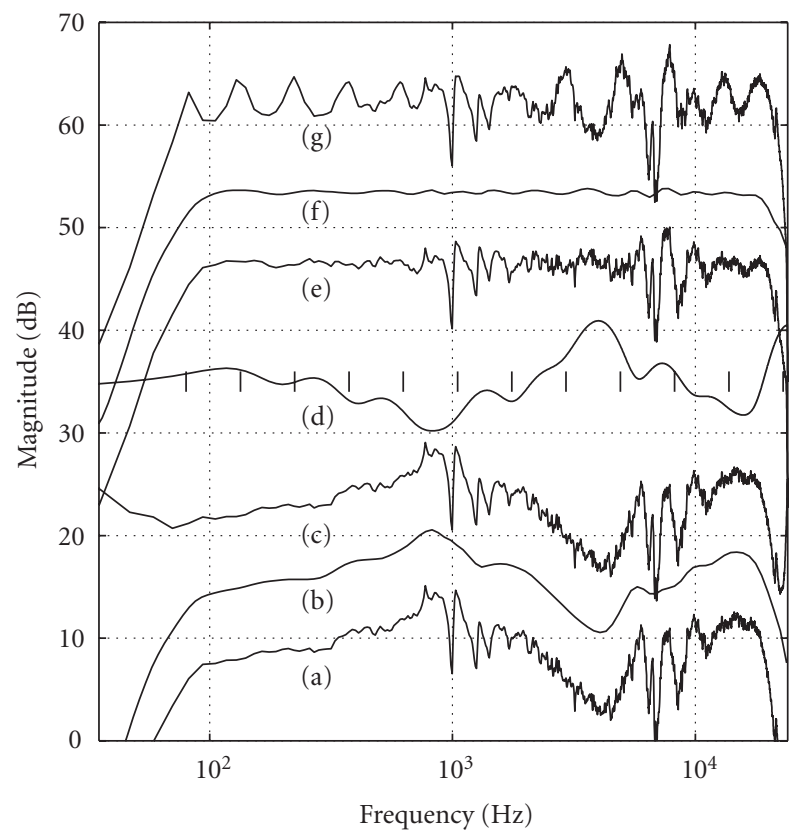

Figure 7: Example of direct LS Kautz EQ. From bottom up: (a) measured magnitude response of loudspeaker; (b) same one 1/3octave smoothed; (c) after low and high roll-off compensation; (d) magnitude response of 24 th-order (12 pole pairs) Kautz equalizer; (e) equalized magnitude response; (f) same one 1/3-octave smoothed; and (g) Kautz EQ response with $R=0.8$. Vertical lines at $35 \mathrm{~dB}$ level indicate logarithmically spaced pole frequencies.

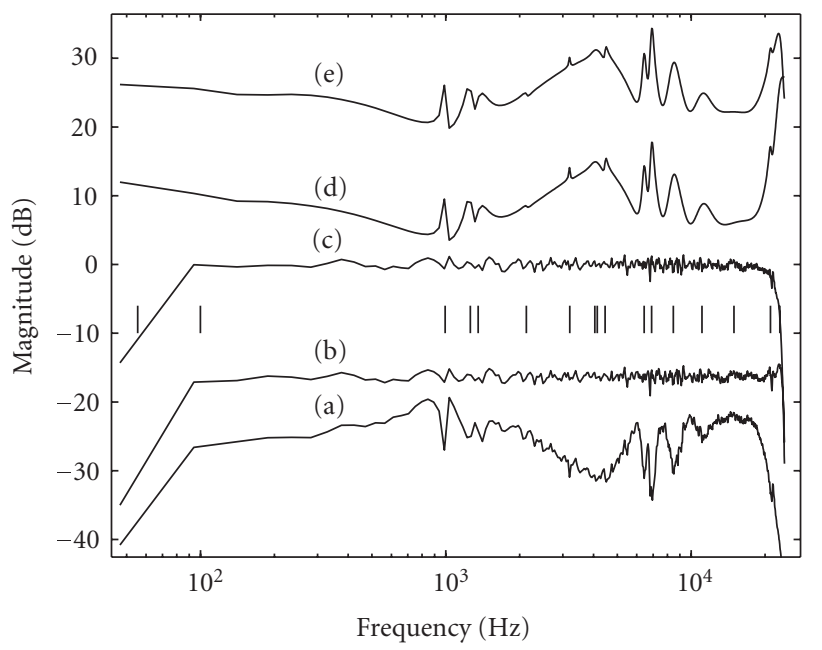

FIGURE 8: Magnitude responses from bottom to top: (a) measured loudspeaker response; (b) direct LS equalized by method (9); pole frequencies; (c) indirect equalized using (5) with respect to inverted target; and (d)-(e) corresponding equalizer responses, respectively.

behavior of an idealized two-way loudspeaker. It consists of a low-frequency driver in a vented box (4th-order highpass at $80 \mathrm{~Hz}$ ) and a high-frequency driver, both with flat response except the low-frequency roll-off. They are combined with 


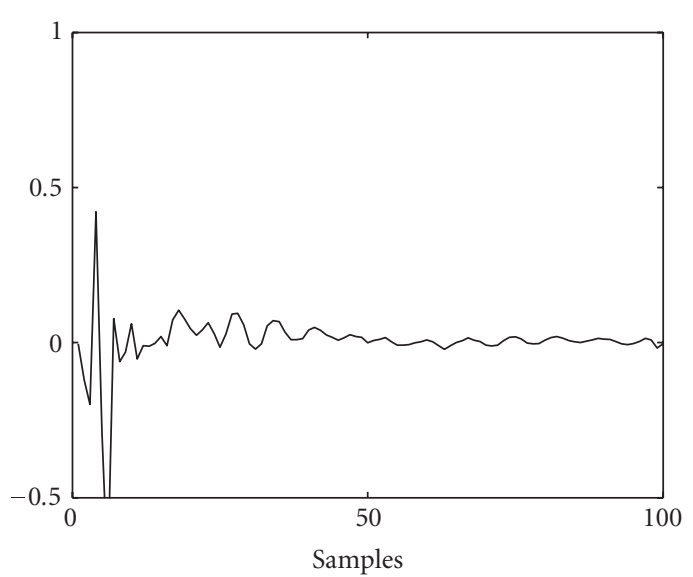

(a)

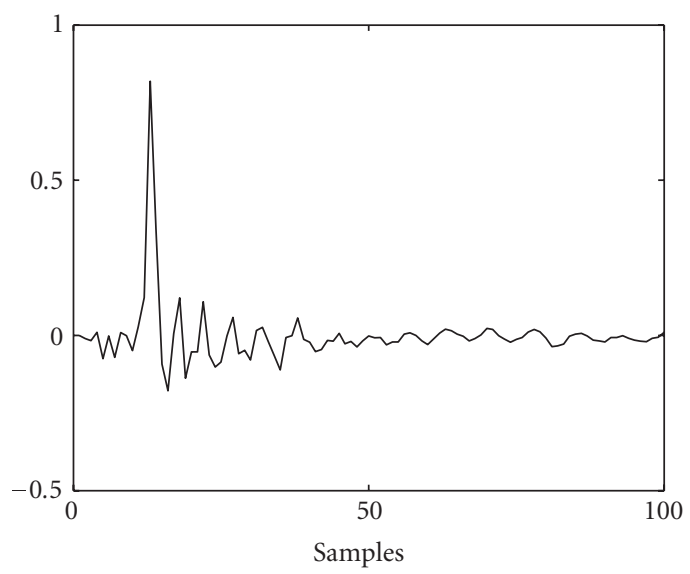

(c)

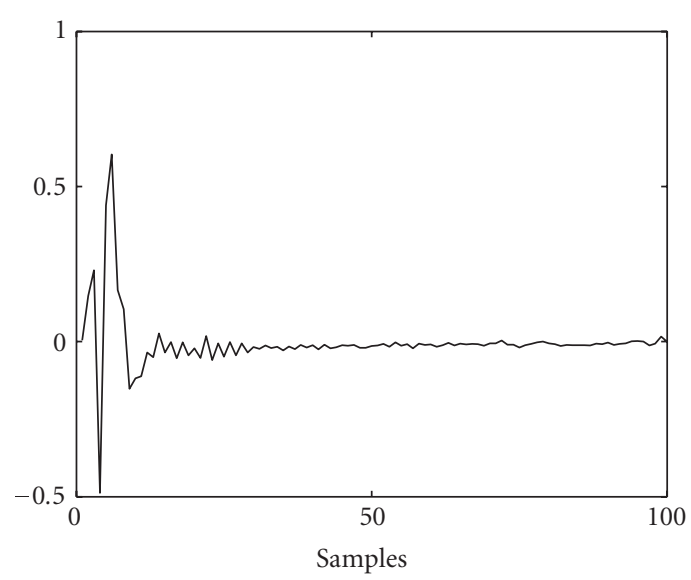

(b)

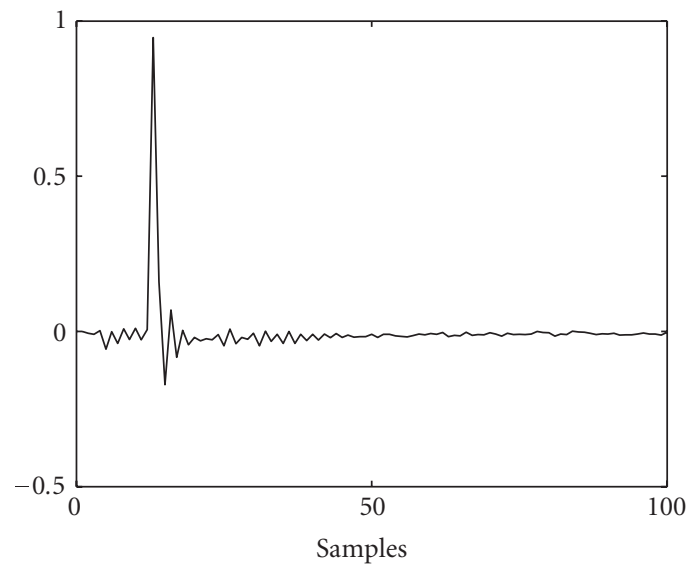

(d)

FIGURE 9: Early part of time-domain responses: (a) measured loudspeaker; (b) LS equalized (Kautz filter order 38); (c) using the same set of poles and including delay $(\Delta=12$ samples) in target; and (d) by including 12 poles at the origin and delay $(\Delta=12$ samples) in target.

a second-order Linkwitz-Riley crossover network [28, 29], which in an ideal case results in a flat magnitude response at the main axis.

In this particular case we investigate a loudspeaker where the acoustic center of the high-frequency driver is $17 \mathrm{~cm}$ behind the acoustic center of the low-frequency unit. This means a temporal nonalignment of about $0.5 \mathrm{~ms}$, which results in ripple of the main axis magnitude response ("Original" in Figure 10) and similarly a nonflat group delay response ("Original" in Figure 11). The magnitude response error of this amount is audible. Although the group delay deviation remains within $1 \mathrm{~ms}$ above $300 \mathrm{~Hz}$, which is hardly noticeable in practice, it is interesting to check how the phase correction by Kautz LS equalization works. This brings necessarily latency beyond the maximum group delay of the original response.

Curves "EQ min-phase" in Figures 10 and 11 show the magnitude and group delay responses of the simulated loudspeaker when a Kautz equalizer is designed based on the minimum-phase part of the loudspeaker impulse response.
The Kautz filter has 18 pole pairs and it was designed with logarithmic distribution of poles between $80 \mathrm{~Hz}$ and $23 \mathrm{kHz}$ and pole radius coefficient $R=0.1$. The low-frequency roll-off is compensated in EQ design to remain as it was originally. After equalization the magnitude response is flat within $\pm 1 \mathrm{~dB}$, while the group delay (dashed line) is not essentially improved (dashed curve in Figure 11).

Curves "EQ excess-phase" in Figures 10 and 11 illustrate the results of magnitude plus phase equalization with a Kautz LS equalizer. In this case the target response of the equalized system is given as a delayed impulse, with a latency higher than the maximum delay of the loudspeaker itself. The target group delay was set here to $1.5 \mathrm{~ms}$ (66 samples at $44.1 \mathrm{kHz}$ sample rate). A direct LS Kautz equalizer was designed with 8 logarithmically distributed pole pairs within $80 \mathrm{~Hz}$ to $23 \mathrm{kHz}$, with $R=0.05$, plus 96 poles at the origin. Notice that the latter ones correspond again to FIR filter behavior, so that the equalizer is a mixture of an FIR and an IIR filter.

After applying excess-phase equalization the magnitude response in Figure 10 is again within $\pm 1 \mathrm{~dB}$, while the 


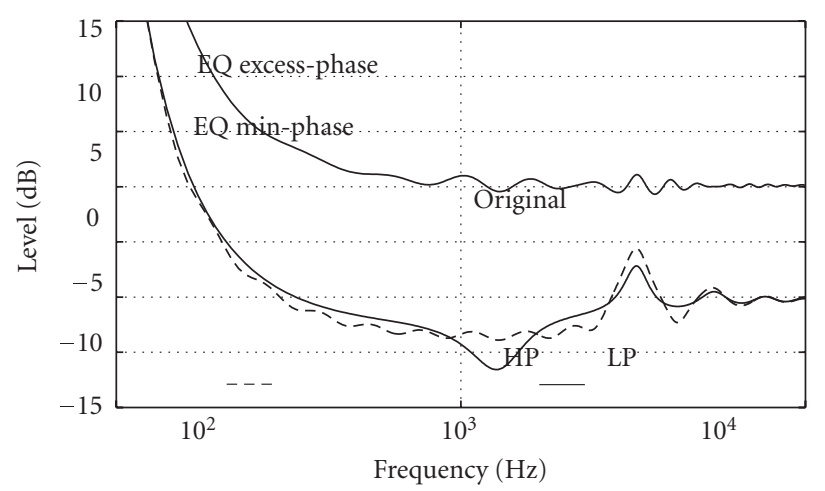

FIGURE 10: Magnitude responses of the simulated loudspeaker: LP = low-pass crossover; HP = highpass crossover; original = response due to driver distance misalignment; minimum-phase equalized; excess-phase equalized response.

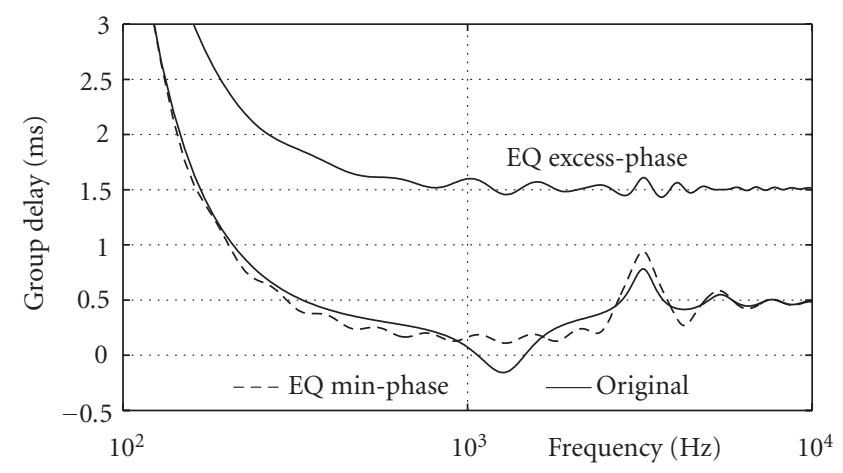

FIGURE 11: Group delay responses of the simulated loudspeaker: original $=$ ripple due to driver distance misalignment; minimumphase equalized; excess-phase equalized response with extra group delay.

group delay curve in Figure 11 has ripple less than $\pm 0.1 \mathrm{~ms}$. (The growth of low-frequency group delay comes from the highpass behavior of the loudspeaker, which is not compensated for.)

Figure 12 plots the time-domain responses of the original simulated loudspeaker, and its minimum-phase and nonminimum-phase versions. Minimum-phase equalization makes the impulse response even worse with some postoscillation, while allowing excess delay in nonminimum-phase design makes the response close to an ideal impulse.

\section{ROOM RESPONSE EQUALIZATION CASES}

In this section we examine two basic examples of room response correction using Kautz LS equalization.

\subsection{Room response equalization, Case 4}

In this case the loudspeaker had a low-frequency roll-off at about $80 \mathrm{~Hz}$, which was compensated in target response design. The room was a listening room of $33 \mathrm{~m}^{2}$ with fairly

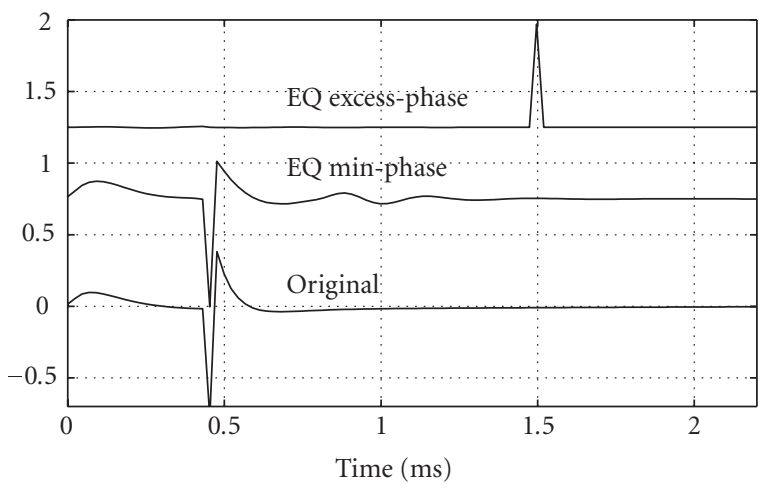

FIGURE 12: Impulse responses of the simulated loudspeaker.

well controlled acoustics. Figure 13 shows the first $5 \mathrm{~ms}$ of the measured impulse response in subplot (a) and magnitude response in subplot (c) in full resolution and 1/3-octave smoothed.

A minimum-phase Kautz equalizer of order 24 (12 pole pairs) was designed with logarithmically positioned pole frequencies between $50 \mathrm{~Hz}$ and $20 \mathrm{kHz}$, using pole radius parameter $R=0.5$. The resulting impulse response and magnitude response are plotted in Figure 13, subplots (b) and (d), respectively. The magnitude response is flattened as desired. In the impulse response some low-frequency oscillation is damped, but the peaks corresponding to reflections from surfaces cannot naturally be canceled out by such a low-order equalizer. Equalizer filter orders down to 8-12 (46 pole pairs) provide useful equalization results in this particular case.

\subsection{Room response equalization, Case 5}

The use of prefixed pole distributions in defining the Kautz equalizer, such as the logarithmic one, can be seen as a "signal-independent" way of reflecting desired overall resolution of modeling. The signal-dependent or case-specific approach would then correspond to approximating a somehow attained inverse target response in a way that also includes optimization of the pole positions. This was done in the loudspeaker equalization Case 2, where the poles were generated with respect to an inverted minimum-phase target response. The same procedure can in principle be applied to the case of room response equalization, although the following example is included mainly as a cautionary and speculative curiosity, demonstrating the capabilities and limitations of Kautz equalization.

Figure 14 displays the magnitude response characteristics of a 320th-order Kautz equalizer. The Kautz filter poles were generated with respect to a DFT-based minimum-phase inverted target response of the measured room response (including compensation of the low-frequency roll-off). The warped BU-method, as described in [27], was used to emphasize the lower frequency region, which in effect also reduces the need for controlling the high end roll-off. 


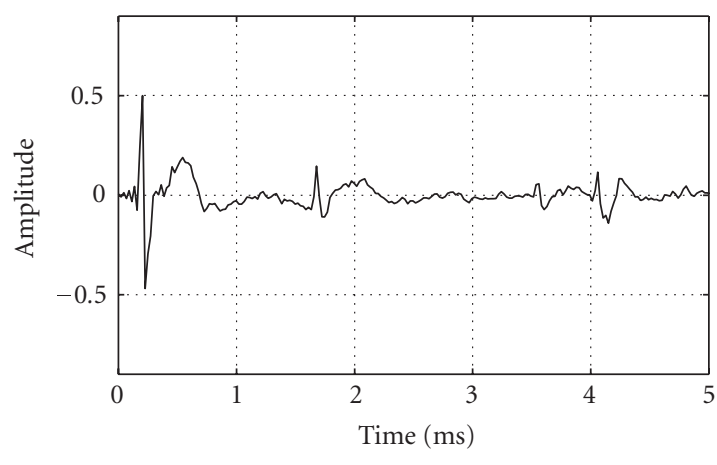

(a)

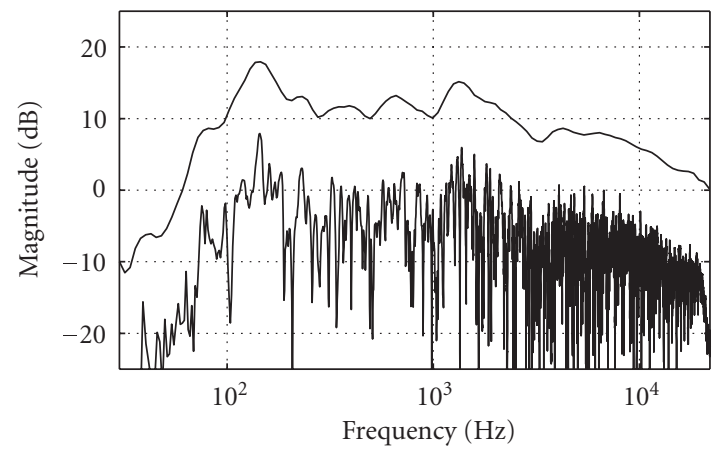

(c)

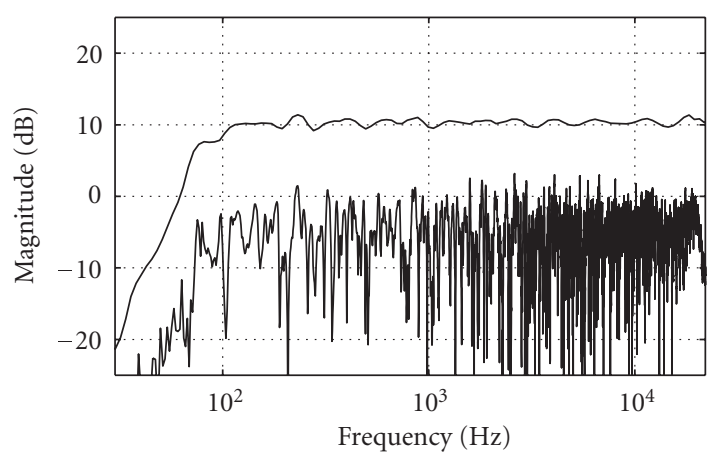

(b)

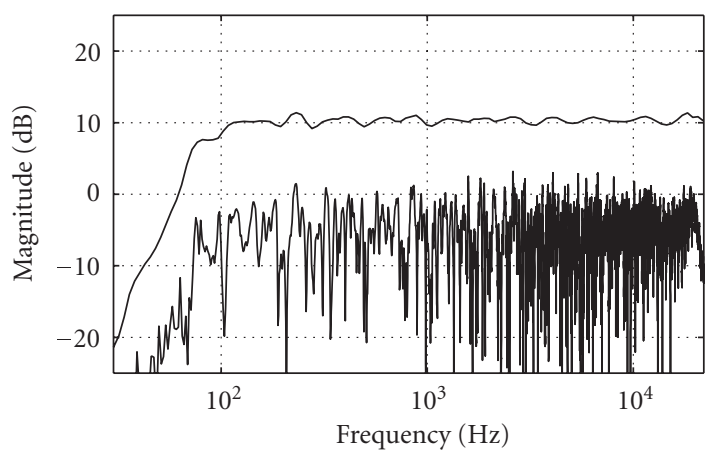

(d)

Figure 13: Kautz equalization of a room response: (a) first $5 \mathrm{~ms}$ of the impulse response; (b) first $5 \mathrm{~ms}$ of the equalized impulse response; (c) original magnitude response; and (d) Kautz equalized magnitude response. In magnitude responses the lifted upper curves are computed through $1 / 3$-octave smoothing.

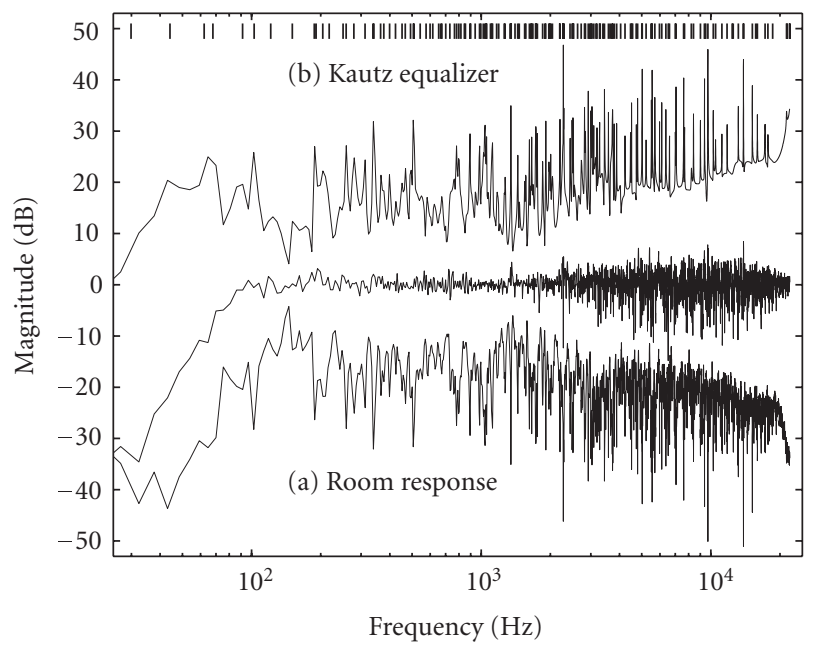

Figure 14: Magnitude response of a 320th-order Kautz equalizer (b) constructed from a minimum-phase inverse of the measured room response (a). The magnitude equalization result is in the middle and the lines on the top indicate Kautz filter pole positions.

This kind of target signal lengths and filter orders are admittedly at the limit of the capabilities of the BU-method, possibly already somewhat on the questionable side. Nevertheless, the pole optimization process seems to be able to find and equalize modal components: in the lower frequency part this could actually in some cases be desirable, but the spiking of the higher frequency part of the equalizer response more or less contradicts the idea of using a Kautz equalizer. It is possible to control the pole set, for example, by direct tuning of the poles or by further modifications of target response in the pole optimization procedure. An interesting possibility would be to use (complex) smoothing techniques [10] as preprocessing for the BU-method.

The question of including perceptually meaningful phase equalization into the Kautz equalizer configuration seems to be particularly difficult in the case of room response equalization. One would hope that adding zeros to the pole set and a corresponding amount of allowed delay for the overall system would result in useful approximative phase response equalization, such as reduced reverberation. This is however apparently not the case: the amount of zeros and combined processing delay has to be increased to the limit of "genuine FIR LS nonminimum-phase equalization" before the magnitude response equalization results regain usability. In fact, one way to proceed is to use a sufficiently high-order nonminimum-phase FIR LS equalizer as the new target for the Kautz equalizer. The initial growing part 
before the maximum amplitude can then be implemented as an FIR substructure of the Kautz equalizer, whereas the decaying part or the equalizer response is approximated as a "forward" Kautz model, for example, by using the BU-method to extract the poles.

Based on our experience so far, the Kautz filters may not offer clear advantages in phase correction of room responses, because the FIR part needed is of such a high order that the IIR part compactness does not help much, and because the design process itself is complex and computationally expensive.

\section{DISCUSSION AND CONCLUSIONS}

In the present study we have extended the use of Kautz filters for loudspeaker and room response correction. The novelty is to apply least squares optimal direct design of Kautz equalizers. Logarithmic frequency resolution is approximated by setting the distribution of pole frequencies logarithmically and by controlling the pole radii to approximate constant$\mathrm{Q}$ behavior. This is conceptually similar to EQ filter design through linear prediction in the case of uniform frequency resolution.

An advantage of the new method is that it can be applied to nonminimum-phase EQ design, as demonstrated for loudspeaker equalization. Another favorable feature is the inherent control of sharp magnitude response dips to avoid corresponding sharp peaks and long-ringing temporal decays in the equalizer. This is done simply by limiting the Qvalues of the Kautz system pole pairs.

Low-order Kautz equalizers ( 4 to 12 pole pairs) are found in practice to yield good magnitude response correction. Phase correction may need higher orders with a set of system poles at the origin and excess delay required for the target response.

Kautz filters are known to be numerically robust due to all pass type of elements used in the implementation. For low orders it is possible to map the EQ transfer function obtained to a direct form IIR structure for maximal efficiency on DSP processors. This and many other practical questions remain however out of the scope of this paper.

A web page with Matlab code examples for Kautz equalization is available at http://acoustics.hut.fi/demos/KautzEQ. html.

\section{ACKNOWLEDGMENT}

The work of Tuomas Paatero has been funded by the Academy of Finland, project no. 205787 (Virtual Acoustics and Audio 2015).

\section{REFERENCES}

[1] R. Greenfield and M. O. Hawksford, "Efficient filter design for loudspeaker equalization," Journal of the Audio Engineering Society, vol. 39, no. 10, pp. 739-751, 1991.

[2] M. Karjalainen, E. Piirilä, A. Järvinen, and J. Huopaniemi, "Comparison of loudspeaker equalization methods based on
DSP techniques," Journal of the Audio Engineering Society, vol. 47, no. 1-2, pp. 15-31, 1999.

[3] M. Karjalainen, T. Paatero, J. N. Mourjopoulos, and P. D. Hatziantoniou, "About room response equalization and dereverberation," in Proceedings of IEEE Workshop on Applications of Signal Processing to Audio and Acoustics (WASPAA '05), pp. 183-186, New Paltz, NY, USA, October 2005.

[4] M. Miyoshi and Y. Kaneda, "Inverse filtering of room acoustics," IEEE Transactions on Acoustics, Speech, and Signal Processing, vol. 36, no. 2, pp. 145-152, 1988.

[5] J. N. Mourjopoulos, "Digital equalization of room acoustics," Journal of the Audio Engineering Society, vol. 42, no. 11, pp. 884-900, 1994.

[6] J. N. Mourjopoulos, "Comments on 'analysis of traditional and reverberation-reducing methods of room equalization," Journal of the Audio Engineering Society, vol. 51, no. 12, pp. 1186-1188, 2003.

[7] S. T. Neely and J. B. Allen, "Invertibility of a room impulse response," Journal of the Acoustical Society of America, vol. 66, no. 1, pp. 165-169, 1979.

[8] B. D. Radlovic and R. A. Kennedy, "Nonminimum-phase equalization and its subjective importance in room acoustics," IEEE Transactions on Speech and Audio Processing, vol. 8, no. 6, pp. 728-737, 2000.

[9] T. Paatero and M. Karjalainen, "Kautz filters and generalized frequency resolution: theory and audio applications," Journal of the Audio Engineering Society, vol. 51, no. 1-2, pp. 27-44, 2003.

[10] P. D. Hatziantoniou and J. N. Mourjopoulos, "Generalized fractional-octave smoothing of audio and acoustic responses," Journal of the Audio Engineering Society, vol. 48, no. 4, pp. 259280, 2000.

[11] J. W. Worley, P. D. Hatziantoniou, and J. N. Mourjopoulos, "Subjective assessments of real-time room dereverberation and loudspeaker equalization," in Proceedings of 118th Audio Engineering Society Convention, Barcelona, Spain, May 2005, Paper 6461.

[12] A. Mäkivirta, P. Antsalo, M. Karjalainen, and V. Välimäki, "Modal equalization of loudspeaker-room responses at low frequencies," Journal of the Audio Engineering Society, vol. 51, no. 5, pp. 324-343, 2003.

[13] L. D. Fielder, "Analysis of traditional and reverberationreducing methods of room equalization," Journal of the Audio Engineering Society, vol. 51, no. 1-2, pp. 3-26, 2003.

[14] T. Paatero and M. Karjalainen, "Equalization of audio systems using Kautz filters with logarithmic allocation of frequency resolution," in Proceedings of 120th Audio Engineering Society Convention, Paris, France, May 2006, Paper 6767.

[15] P. W. Broome, "Discrete orthonormal sequences," Journal of the Association for Computing Machinery, vol. 12, no. 2, pp. 151-168, 1965.

[16] W. H. Kautz, "Transient synthesis in the time domain," IRE Transactions on Circuit Theory, vol. 1, pp. 29-39, 1954.

[17] J. L. Walsh, Interpolation and Approximation by Rational Functions in the Complex Domain, American Mathematical Society, Providence, RI, USA, 2nd edition, 1969.

[18] J. Mourjopoulos, P. Clarkson, and J. Hammond, "A comparative study of least-squares and homomorphic techniques for the inversion of mixed phase signals," in Proceedings of IEEE International Conference on Acoustics, Speech, and Signal Processing (ICASSP '82), vol. 7, pp. 1858-1861, Paris, France, May 1982. 
[19] A. Härmä, M. Karjalainen, L. Savioja, V. Välimäki, U. K. Laine, and J. Huopaniemi, "Frequency-warped signal processing for audio applications," Journal of the Audio Engineering Society, vol. 48, no. 11, pp. 1011-1031, 2000.

[20] B. C. J. Moore, R. W. Peters, and B. R. Glasberg, "Auditory filter shapes at low center frequencies," Journal of the Acoustical Society of America, vol. 88, no. 1, pp. 132-140, 1990.

[21] A. Härmä and T. Paatero, "Discrete representation of signals on a logarithmic frequency scale," in Proceedings of IEEE Workshop on Applications of Signal Processing to Audio and Acoustics (WASPAA '01), pp. 39-42, New Paltz, NY, USA, October 2001.

[22] T. W. Parks and C. S. Burrus, Digital Filter Design, John Wiley \& Sons, New York, NY, USA, 1987.

[23] M. H. Hayes, Statistical Digital Signal Processing and Modeling, John Wiley \& Sons, New York, NY, USA, 1996.

[24] T. Y. Young and W. H. Huggins, "Complementary' signals and orthogonalized exponentials," IRE Transactions on Circuit Theory, vol. 9, pp. 362-370, 1962.

[25] H. Brandenstein and R. Unbehauen, "Least-squares approximation of FIR by IIR digital filters," IEEE Transactions on Signal Processing, vol. 46, no. 1, pp. 21-30, 1998.

[26] T. Paatero, "Generalized linear-in-parameter models-theory and audio signal processing applications," Doctoral thesis, Helsinki University of Technology, Laboratory of Acoustics and Audio Signal Processing (Report no. 75), Espoo, Finland, November 2005.

[27] T. Paatero, "An audio motivated hybrid of warping and Kautz filter techniques," in Proceedings of European Signal Processing Conference (EUSIPCO '02), pp. 627-630, Toulouse, France, September 2002.

[28] S. H. Linkwitz, "Active crossover networks for noncoincident drivers," Journal of the Audio Engineering Society, vol. 24, no. 1, pp. 2-8, 1976.

[29] S. H. Linkwitz, "Passive crossover networks for noncoincident drivers," Journal of the Audio Engineering Society, vol. 26, no. 3, pp. 149-150, 1978.

Matti Karjalainen was born in Hankasalmi, Finland, in 1946. He received M.S. and Dr.Sc. (Tech.) degrees in electrical engineering from the Tampere University of Technology, in 1970 and 1978, respectively. From 1980 he is Professor in acoustics and audio signal processing at the Helsinki University of Technology in the Department of Electrical and Communications Engineering. In audio technology his main interest is

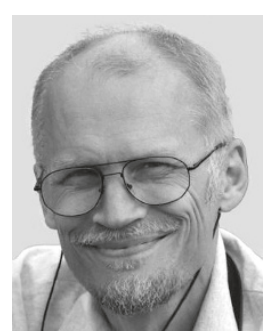
in audio signal processing, such as DSP for sound reproduction and auralization, music DSP and sound synthesis, as well as perceptually based signal processing. In addition to audio DSP his research activities cover speech processing, perceptual auditory modeling and spatial hearing, DSP hardware, software, and programming environments, as well as various branches of acoustics, including musical acoustics and modeling of musical instruments. He has written 350 scientific and engineering papers and contributed to organizing several conferences and workshops. He is an AES Fellow and Silver Medalist as well as Member of IEEE (Institute of Electrical and Electronics Engineers), ASA (Acoustical Society of America), EAA (European Acoustics Association), ISCA (International Speech Communication Association), and several Finnish scientific and engineering societies.
Tuomas Paatero was born in Helsinki, Finland, in 1969. He received in mathematics and physics at the University of Helsinki, M.S. degree in 1994. His more recent postgraduate studies at the Helsinki University of Technology (TKK) were oriented to acoustics and audio signal processing, receiving the Lic.Tech. and Dr.Sc.(Tech.) degrees in 2002 and 2005, respectively. As a Researcher in the Laboratory of Acoustics

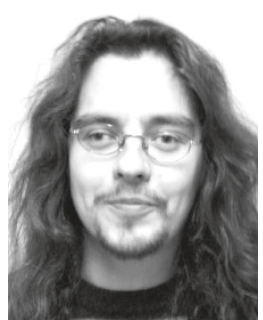
and Audio Signal Processing, TKK, his current research interests include rational orthonormal model and filter structures and their audio signal processing applications, as well as more general considerations on mathematical means in DSP. 